\title{
Imaging through turbulence using compressive coherence sensing
}

\author{
Ashwin A. Wagadarikar, Daniel L. Marks, Kerkil Choi, Ryoichi Horisaki, and David J. Brady* \\ Department of Electrical and Computer Engineering, Duke University, Durham, North Carolina 27708, USA \\ *Corresponding author: dbrady@duke.edu
}

Received December 3, 2009; revised February 4, 2010; accepted February 5, 2010; posted February 19, 2010 (Doc. ID 120492); published March 12, 2010

\begin{abstract}
Previous studies have shown that the isoplanatic distortion due to turbulence and the image of a remote object may be jointly estimated from the $4 \mathrm{D}$ mutual intensity across an aperture. This Letter shows that decompressive inference on a $2 \mathrm{D}$ slice of the $4 \mathrm{D}$ mutual intensity, as measured by a rotational shear interferometer, is sufficient for estimation of sparse objects imaged through turbulence. The $2 \mathrm{D}$ slice is processed using an iterative algorithm that alternates between estimating the sparse objects and estimating the turbulence-induced phase screen. This approach may enable new systems that infer object properties through turbulence without exhaustive sampling of coherence functions. () 2010 Optical Society of America OCIS codes: $110.1650,110.3175,280.7060$.
\end{abstract}

Atmospheric turbulence distorts the wavefronts from incoherent sources radiating in the far field so that imaging the sources without compensating for the distortion results in a degraded image. Distorted wavefronts may be characterized by wavefront sensors [1] to control the actuators of a deformable mirror in an adaptive optics system so that the wavefront aberrations may be removed. After characterization, the distorted wavefront may also be used to digitally compensate the degraded images [2] Alternatively, phase diversity [3] may be used to digitally estimate both the scene and the turbulenceinduced aberrations.

Another strategy relies on measurement of the mutual intensity, a 4D function that captures the correlations between every pair of points in a $2 \mathrm{D}$ aperture. For example, the astigmatic coherence sensor [4], which may be viewed as a generalized phase diversity sensor, samples the 4D mutual intensity of the distorted optical field from remote sources, and uses the coherent-mode decomposition technique to correct an isoplanatic refractive distortion in the pupil. This approach is slow and impractical for image formation, as it requires complete sampling of the mutual intensity.

We propose an approach inspired by compressive sampling (CS) [5] to digitally process a limited subset of samples of the mutual intensity and jointly recover (i) an unaberrated distribution of incoherent sources radiating in the far field and (ii) the phase screen introduced by turbulence that distorts the propagating wavefronts.

The mutual intensity is a 4D function describing the coherence of the optical field and is given by

$$
J\left(\overline{\mathbf{r}}-\frac{\Delta \mathbf{r}}{2}, \overline{\mathbf{r}}+\frac{\Delta \mathbf{r}}{2}\right)=\left\langle E\left(\overline{\mathbf{r}}-\frac{\Delta \mathbf{r}}{2}\right)^{*} E\left(\overline{\mathbf{r}}+\frac{\Delta \mathbf{r}}{2}\right)\right\rangle,
$$

where $E(\mathbf{r})$ is a quasi-monochromatic, statistically wide sense stationary electromagnetic field of wavenumber $k=\omega / c$ and $\overline{\mathbf{r}}$ is the mean position of a pair of coordinates separated by $\Delta \mathbf{r}$. The van CittertZernike (VCZ) theorem [6] states that $J$ in a $2 \mathrm{D}$ ap- erture in the far field of spatially incoherent sources with irradiance $S(\mathbf{r})$ depends only on $\Delta \mathbf{r}$ as

$$
J(\Delta \mathbf{r})=\int \frac{S(\mathbf{r})}{z^{2}} e^{-i \omega / c z(\Delta \mathbf{r} \cdot \mathbf{r})} d^{2} \mathbf{r} .
$$

Thus, $J$ is the 2D Fourier transform of the scene. To image the scene, $J$ may be sampled as a function of $\Delta \mathbf{r}$ about a central point using an interferometer and then inverse Fourier transformed.

If isoplanatic turbulence with an optical path delay, $d(\mathbf{r})$, is present between the scene and the aperture,

$$
\begin{aligned}
J\left(\overline{\mathbf{r}}-\frac{\Delta \mathbf{r}}{2}, \overline{\mathbf{r}}+\frac{\Delta \mathbf{r}}{2}\right)= & {\left[\int \frac{S(\mathbf{r})}{z^{2}} e^{-i \omega / c z[(\Delta \mathbf{r}) \cdot \mathbf{r}]} d^{2} \mathbf{r}\right] } \\
& \times e^{i \omega / c[d(\overline{\mathbf{r}}-\Delta \mathbf{r} / 2)-d(\overline{\mathbf{r}}+\Delta \mathbf{r} / 2)]} .
\end{aligned}
$$

Note that $J$ is now a function of both $\overline{\mathbf{r}}$ and $\Delta \mathbf{r}$. Thus, when turbulence is present, directly applying the VCZ theorem described in Eq. (2) produces a distorted image of $S(\mathbf{r})$. Accurate recovery of $S(\mathbf{r})$ would normally require knowledge of the complete $4 \mathrm{D}$ distribution of $J$.

A rotational shear interferometer (RSI) [6], as shown in Fig. 1, can be used to measure samples of $J$. In this instrument, a beam splitter divides the optical field to be sampled at the aperture into two cop-

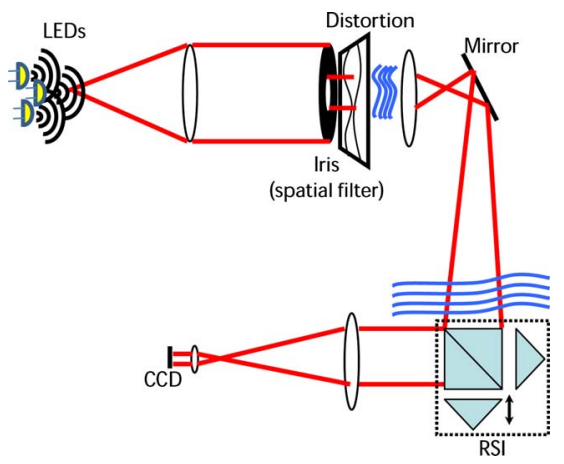

Fig. 1. (Color online) Experimental setup to measure the mutual intensity from LEDs with a phase distortion. 
ies, and each copy is reflected by a folding mirror. The orientations of the fold axes of the two mirrors with respect to the $x$ axis are $\theta$ and $-\theta$, respectively. Samples of the complex phase-resolved $J$ can be isolated from quadrature phase-shifted intensity measurements on the RSI detector array acquired by changing the optical path length, $l$, of one arm of the RSI using a piezo stage. At pixel $(x, y)$ on the $2 \mathrm{D}$ detector, we recover

$$
\begin{aligned}
J(x, y)= & {\left[\int \frac{S\left(x^{\prime}, y^{\prime}\right)}{z^{2}} e^{2 i \omega / c z\left(y x^{\prime}+x y^{\prime}\right) \sin (2 \theta)} \mathrm{d} x^{\prime} \mathrm{d} y^{\prime}\right] } \\
& \times e^{i \varphi(x, y)},
\end{aligned}
$$

where $\varphi(x, y)=\omega / c[d(\overline{\mathbf{r}}-\Delta \mathbf{r} / 2)-d(\overline{\mathbf{r}}+\Delta \mathbf{r} / 2)]$, with $\overline{\mathbf{r}}$ $=(-x \cos (2 \theta), y \cos (2 \theta)) \quad$ and $\Delta \mathbf{r}=(-2 y \sin (2 \theta)$, $-2 x \sin (2 \theta))$. This measurement represents a sample of a rotated and scaled version of the $2 \mathrm{D}$ mutual intensity in Eq. (2) that is modulated by a $2 \mathrm{D}$ turbulence-induced phase screen, $\varphi(x, y)$, in the RSI aperture. In the absence of turbulence, the measured samples have Hermitian symmetry, i.e., $J(x, y)$ $=J(-x,-y)^{*}$, since they represent a Fourier transformation of a real function, the irradiance of the scene, $S\left(x^{\prime}, y^{\prime}\right)$. If the angle $\theta \neq \pi / 2$, in the presence of turbulence, the samples do not have Hermitian symmetry. Because of this disparity, the turbulence phase can be distinguished from the phase owing to the scene itself.

We show that it is possible to jointly recover the scene and the phase screen from just a $2 \mathrm{D}$ subset of $J$ that is measured by an RSI. Mathematically, solving this problem can be posed as

$$
\left[x_{e}, P_{e}\right]=\underset{x, P}{\arg \min }\|g-A P F x\|_{2}^{2},
$$

where $x_{e}$ is a discrete estimate of the 2D scene $S\left(x^{\prime}, y^{\prime}\right), P_{e}$ is a discrete estimate of the turbulenceinduced phase screen $\varphi(x, y), g$ is a vector of the $2 \mathrm{D}$ subset of samples of $J$ measured on the RSI, $A$ is a system-specific operator that places more weight on the lower spatial frequencies in the image, and $F$ is a 2D discrete Fourier transform (DFT) matrix. Since $P$ and $x$ are both unknown, this is a nonconvex problem, which implies that a globally optimal solution cannot be found efficiently. Our approach to this joint recovery problem relies on iterative alternating minimization [7] and CS theory to converge to a local minimum of (5). Applying CS ideas requires us to assume as prior knowledge that the scene being imaged through turbulence is sparse on some basis.

In a nutshell, CS theory can accurately decode some discrete signal, $x$, sparse in some basis $\Psi$ with basis coefficients $f$, from a limited number of multiplexed measurements, $g$, generated by $\Phi ; g=\Phi x$ $=\Phi \Psi f$. Let an $S$-sparse signal $x \in \mathbb{R}^{N}$ be defined as having exactly $S$ nonzero basis coefficients and $(N$ $-S)$ zero coefficients. An essence of CS is that an $S$-sparse $x$ can be accurately reconstructed with high probability by solving

$$
f_{e}=\arg \min _{f}\|f\|_{1} \text { s.t. } g=\Phi x_{e}=\Phi \Psi f_{e}=H f_{e},
$$

provided that $H$ satisfies known sufficient conditions [5].

We use these concepts to experimentally demonstrate imaging of point sources in a scene through isoplanatic turbulence with merely a $2 \mathrm{D}$ subset of the $4 \mathrm{D} J$, as measured by the RSI. In our case, the signal of interest is a sparse scene, which can be discretely represented as $x=I f$. The operator $H=\Phi \Psi=\Phi I=\Phi$ describes the propagation of spatially partially coherent light from the incoherent point sources by the VCZ theorem including turbulence. Our problem is complicated by the fact that the phase screen is unknown, which implies that $H=\Phi$ is not fully known.

Consequently, our approach to the problem of joint recovery of the phase screen and a sparse scene, as posed in Eq. (5), is an iterative two-step alternating minimization technique. In the first step, a CS algorithm is used to estimate an undistorted and sparse distribution of sources in the scene that fits the measurements of $J$ as closely as possible, given an estimate of the phase screen. In the other step, the phase screen estimate is refined using the measurements of $J$ on the RSI and a progressively undistorted version of $J$ that is modeled using the current estimate of the sparse scene. These two steps are repeated until there is minimal change in the estimate of the sparse scene. Mathematically, the $i$ th iteration of this process is represented as

$$
\begin{aligned}
x_{e}^{i} & =\underset{x}{\arg \min } \frac{1}{2}\|g-\Phi x\|_{2}^{2}+\lambda\|x\|_{1} \\
& =\underset{x}{\arg \min } \frac{1}{2}\left\|g-A P_{e}^{i-1} F x\right\|_{2}^{2}+\lambda\|x\|_{1} \\
P_{e}^{i} & =\operatorname{sgn}\left[\left(A F x_{e}^{i}\right)^{*} \cdot g\right],
\end{aligned}
$$

where $x_{e}^{i}$ and $P_{e}^{i}$ are the estimates of the scene and the phase screen at the $i$ th iteration to be recovered from $g$, a vector of samples of the measured $J$. Equation (7a) is the Lagrangian unconstrained optimization formulation of Eq. (6), with $\lambda$ as a regularization parameter. We adapt the two-step iterative shrinkage and thresholding algorithm [8] to solve this illconditioned problem. Equation (7b) is the leastsquares solution to $g=A X p$, where the matrix product $X$ is a diagonal matrix listing the DFT coefficients of $x_{e}^{i}$ on the diagonal and $p$ is a vector of the elements of $P_{e}^{i}$.

To experimentally verify the utility of this approach, a sparse scene consisting of three lightemitting diodes (LEDs) was arranged and was observed through an aberrating phase distortion using the RSI, as detailed in Fig. 1. The LEDs emitted light over $590 \pm 30 \mathrm{~nm}$ (coherence length $=5.8 \mu \mathrm{m})$ and had their plastic lenses sanded off to produce mutually incoherent radiation over $2 \pi$ sr. These LEDs were collimated by a lens to place their image at infinity as would occur for stellar imaging. An iris was used to eliminate vignetting effects from the collimation lens. 
A phase distortion plate was placed behind the iris to produce an instance of the phase distortion caused by turbulence. This plate was constructed by curing polydimethylsiloxane onto a microscope slide using a heat gun. A second lens relayed the partially coherent aberrated field to the RSI aperture. Intensity interferograms were collected as the delay between the two arms of the interferometer was varied. The interferograms at four delays $I_{0}, I_{\pi / 2}, I_{\pi}, I_{3 \pi / 2}$ with phases separated by $\pi / 2$ were summed to find a $2 \mathrm{D}$ subset of the mutual intensity using the formula $J=I_{0}+i I_{\pi / 2}$ $-I_{\pi}-i I_{3 \pi / 2}$. The measurements were then processed using the algorithm described in Eq. (7).

Figure 2(a) shows the magnitude of the 2D subset of samples of $J$ measured using the RSI. Figure 2(b) shows a distorted image of the scene recovered using the VCZ theorem, which does not account for turbulence. Figures 2(c) and 2(d) show discrete estimates of the undistorted scene, $x_{e}$, and the phase screen, $\arg \left\{P_{e}\right\}$, recovered using the algorithm in Eqs. (7). The recovered image of the three LEDs is a clear improvement over the aberrated image in Fig. 2(b).

Figure 3 shows the results of a control experiment without the phase distortion between the LEDs and the RSI. Figure 3(a) shows magnitude of the 2D subset of samples of $J$ measured using the RSI. Figure $3(\mathrm{~b})$ shows an image of the scene recovered using the VCZ theorem. Figures 3(c) and 3(d) show discrete estimates of the scene, $x_{e}$, and the phase screen, $\arg \left\{P_{e}\right\}$, recovered using the algorithm in Eqs. (7). With the use of the sparsity constraint, the algorithm sharpens the images of the three LEDs in the scene. As expected, the phase screen is uniform owing to the lack of turbulence. The control experiment suggests that the algorithm does not merely deconvolve the image directly but estimates the phase screen and applies it to the sampled $J$ to produce an improved image. Comparing Figs. 2(c) and 3(c), we note that the absolute positions of the LEDs are not recovered. This is because the algorithm is unable to recover the

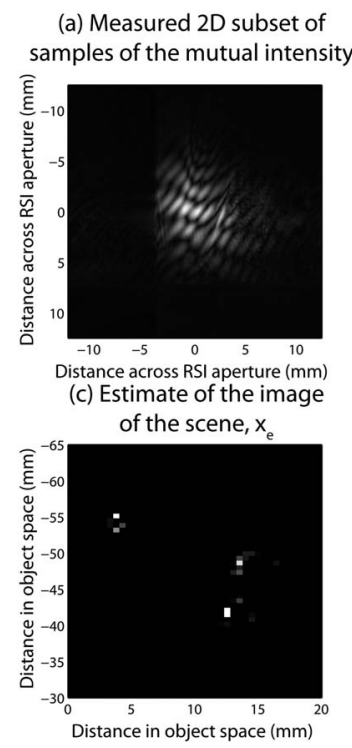

(b) Image of the scene using the VCZ theorem

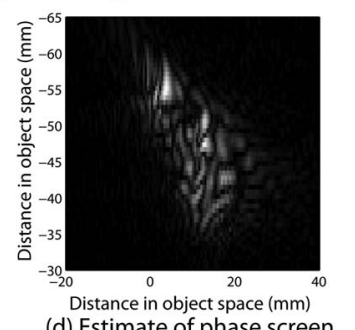

(d) Estimate of phase screen

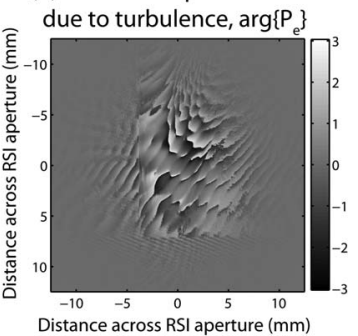

Fig. 2. Results of using approach in Eqs. (7) to image three LEDs aberrated by turbulence.

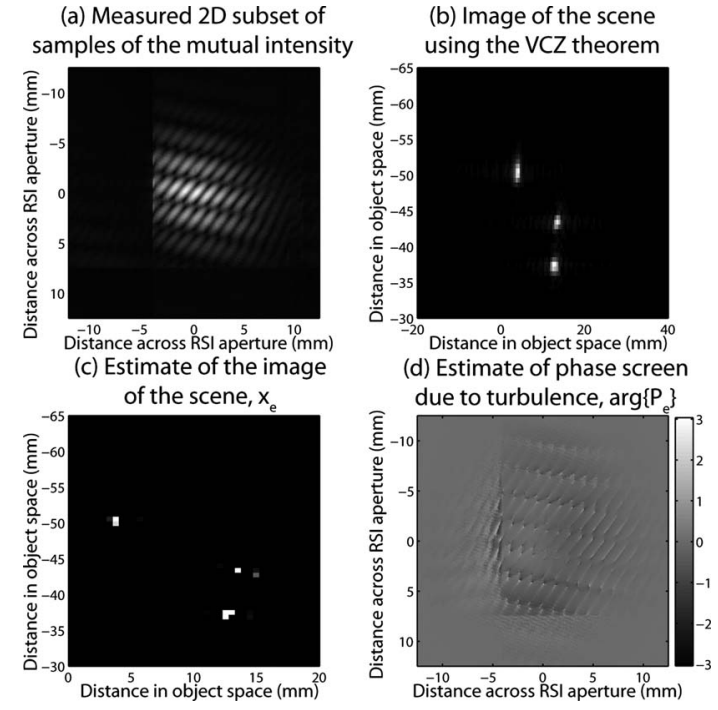

Fig. 3. Results of a control experiment for the reconstruction of three LEDs not aberrated by turbulence.

tilt component of the phase screen that leads to a relative shift in the position of the objects with respect to their true positions.

Noiseless MATLAB simulations suggest that using the RSI to image extended objects through turbulence may also be possible by exploiting sparsity in the spatial gradient of the scene and using a minimum total-variation constraint rather than an $l_{1}$ constraint on the scene. Unfortunately, the signal-tonoise ratio of the RSI is known to degrade as a function of the number of independent source channels in the scene [9]. The proposed approach can be a practical means of imaging through turbulence in astronomy if the complex $J$ can be sampled in a snapshot. This can potentially be achieved using the quadrature phase-shifting interferometer [10], which measures four phase-shifted interferograms in quadrature in a snapshot.

This work was supported by the Air Force Office of Scientific Research contract FA9550-08-1-0151 and the Natural Sciences and Engineering Research Council of Canada.

\section{References}

1. R. Lane and M. Tallon, Appl. Opt. 31, 6902 (1992).

2. J. Primot, G. Rousset, and J. Fontanella, J. Opt. Soc. Am. A 7, 1598 (1990).

3. R. G. Paxman, T. J. Schulz, and J. R. Fienup, J. Opt. Soc. Am. A 9, 1072 (1992).

4. D. Marks, R. Stack, and D. Brady, Appl. Opt. 41, 6050 (2002).

5. E. J. Candes, J. K. Romberg, and T. Tao, Commun. Pure Appl. Math. 59, 1207 (2006).

6. D. Marks, R. Stack, and D. Brady, Appl. Opt. 38, 1332 (2002).

7. S. Boyd and L. Vandenberghe, Convex Optimization (Cambridge U. Press, 2004).

8. J. M. Bioucas-Dias and M. A. T. Figueiredo, IEEE Trans. Image Process. 16, 2992 (2007).

9. S. Basty, M. Neifeld, D. Brady, and S. Kraut, Opt. Commun. 228, 249 (2003).

10. B. Kern, P. Dimotakis, C. Martin, D. Lang, and R. Thessin, Appl. Opt. 44, 7424 (2005). 